\title{
Allergen Immunotherapy: Biomarkers and Clinical Outcome Measures
}

This article was published in the following Dove Press journal:

Journal of Asthma and Allergy

\author{
Constantinos Pitsios \\ Allergy Outpatient Clinic, Medical \\ School, University of Cyprus, Nicosia, \\ Cyprus
}

\begin{abstract}
Clinical trials for allergen immunotherapy products' development and approval are conducted, aiming to monitor safety and efficacy of them. Symptom scores and the use of rescue medication are the primary clinical endpoints used in the conducted clinical trials, while Quality of Life scores and symptom-free days are measurements also used as secondary endpoints. Although the use of in vitro biomarkers might have been more practical and objective, there are yet no broadly used reliable ones accurately reflecting the clinical effects of allergen immunotherapy. On the contrary, in vivo biomarkers, such as the nasal allergy provocation test, are reliable and successfully used. The aim of this review is to describe how to adapt and use biomarkers and clinical outcomes in the everyday practice of Allergists who perform allergen immunotherapy.
\end{abstract}

Keywords: allergic immunotherapy, clinical trials, allergic rhinitis, asthma, biomarkers

\section{Introduction}

Allergen immunotherapy (AIT) was introduced more than a century ago and is recognized as the only immune-modifying causal treatment for respiratory allergies and venom hypersensitivity. ${ }^{1-3}$ As far as desensitization to airborne allergens is regarded, amelioration of respiratory symptoms, prevention of the onset of new sensitizations and sustained effect after completion of AIT are some of the benefits that AIT offers. ${ }^{2}$

Clinical trials have been conducted to examine safety and efficacy of AIT. The first controlled trial supporting AIT's efficacy was published in 1954 and the clinical outcome measures used to evaluate its efficacy were the decrease of symptoms and patients' overall impression on treatment. ${ }^{4}$ Ever since, controlled and randomized clinical trials and some meta-analyses have confirmed AIT's safety and efficacy, with methodological evaluation based mainly on measures of clinical outcomes. 5,6

Symptom scores and the intake of rescue medication are recommended as primary endpoints for AIT trials, while data like scores of "Quality of Life" (QoL) and symptom-free days can serve as secondary endpoints. ${ }^{6,7}$ Clinical trials have shown that both subcutaneous (SCIT) and sublingual (SLIT) modalities of AIT are effectively for allergic rhinitis. ${ }^{2}$ Additional routes of AIT that have more recently been generated are the epicutaneous (EPIC) and the intralymphatic (ILIT) ones. ${ }^{8}$ Based on clinical outcomes, EPIC has shown modest benefit, while contradictory results have been published for the more promising ILIT. ${ }^{8-10}$
Correspondence: Constantinos Pitsios Allergy Outpatient Clinic, Medical School, University of Cyprus, P.O. Box 20537

Nicosia, 1678, Cyprus

Tel +306974348638

Fax +357 22895396

Email pitsios.constantinos@ucy.ac.cy 
Patients participating in the procedure of clinical trials are voluntarily registering the indicated clinical data or are answering to questionnaires. In order to have reliable data, validated questionnaires have been used or, since real-life monitoring mHealth technology has been developed, e-diaries can replace the use of classic daily diaries, rendering the procedure easier and more appealing. ${ }^{11}$

The fact that self-reported data of symptom scores are partially subjective - affected by the personal perception of symptoms' severity or the extent of personal satisfaction on treatment's outcomes - may represent a flaw of clinical trials. Different patients may estimate symptoms or the need for rescue medication in a different way, so a patient's "personality" is reflected in medication scores. By combining these measures with objective parameters, like pollen-count, researchers can empower the impact of clinical outcomes. ${ }^{12}$

The use of appropriate biomarkers, as quantitative measurements, would be an ideal objective assessment in AIT. The use of such biomarkers would offer advantages like the identification of "good-responders" to AIT, definition of the appropriate duration of treatment, prediction of clinical outcomes, monitoring of the treatment effects or even the need for a booster AIT. ${ }^{13,14}$ Although many studies have addressed the underlying immunological mechanisms of AIT and potential in vitro biomarkers, up to now none has been shown to be predictive of the clinical outcomes. ${ }^{15}$

In real-life an allergist needs outcome measures of AIT that may indicate certain decision points (eg, change of extract, extension of treatment) and assist on patients' adherence. An overview of biomarkers, clinical markers and clinical endpoints and the way they can be used in daily practice are described in the present article.

\section{In vitro Biomarkers}

Studies of in vitro biomarkers are based on the knowledge of cellular and humoral events that take place during AIT; these have been grouped and described by Akdis and Akdis in four stages. ${ }^{16}$ The earliest effect of AIT is the decreased mast cell and basophil activity, that do not degranulate although exposed to gradually increased doses of the antigen. The second stage is the generation of allergen-specific Treg and Breg cells and suppression of allergen-specific effector T-cells. The third is the phase of a decrease in specific $\operatorname{IgE}$ and an increase in specific $\operatorname{IgG}_{4}$ levels. Finally, in a frame of years, decreased tissue mast cells and eosinophils with decreased skin test reactivity are observed. ${ }^{16}$
A Task Force of the European Academy of Allergy and Clinical Immunology (EAACI) has described seven domains of potential in vitro biomarkers, connected to humoral and cellular effects of AIT, as well as in vivo biomarkers. ${ }^{17}$ Allergists are familiar with the use of specific IgE (sIgE) and total IgE (tIgE) and besides diagnostic purposes they often try to use them as endpoints of AIT, too. With the exception of these humoral biomarkers, assays that are not broadly available, or are still at an experimental level, are required for the monitoring of most in vitro biomarkers.

Humoral biomarkers include tIgE, sIgE, subclasses of IgG and IgE-blocking factor. Although sIgE and SPT are used in everyday practice, they are not validated monitoring tools of AIT's outcomes. The initial increase of sIgE levels followed by a decrease over time is not connected with relevant changes in symptoms. A high sIgE/tIgE ratio has been proposed as a predictive marker for successful AIT; however, its utility has not been properly evaluated and validated as a predictive biomarker. ${ }^{17,18}$

AIT is causing an increase in concentrations of $\operatorname{IgG}$ subclasses; IgG1 and an even more pronounced one in IgG4. ${ }^{19,20}$ Thanks to their function as blocking antibodies for IgE-mediated basophil/mast cell activation and antigen presentation, IgG4 have been studied as biomarkers of successful AIT. ${ }^{21,22}$ The continuing upward trend of sIgG4 during the course of AIT has been related to an improvement of symptoms. ${ }^{19,23}$ However, absolute levels of IgG4 often fail to correlate with clinical tolerance, likewise absolute levels of SIgE are similarly poorly predictive of disease severity. ${ }^{24}$ Clinical improvement and increase of sIgG4 are two outcomes related to the duration of AIT, but a solid correlation to each other is missing. ${ }^{25,26}$

AIT-induced IgG antibodies are a component of the serum inhibitory activity for IgE, called IgE-blocking factor (IgE-BF). IgE-BF is considered the effect of several factors blocking IgE-allergen binding and inhibiting IgEfacilitated allergen presentation by $\mathrm{B}$ cells to an allergen specific Th2 cell clone. ${ }^{20,27-29}$ An IgE-Facilitated Allergen Binding (IgE-FAB) assay has been developed, representing an in vitro model of facilitated allergen presentation. ${ }^{30}$ Functional assays of inhibitory IgG4 and IgE-BF may be more useful surrogates of clinical response than $\operatorname{IgG} 4 .{ }^{17,30}$ The problem in using these in vitro methods is that the IgE-BF assay is no longer available, while IgE-FAB performed with flow cytometry - is not widely used. ${ }^{17}$

Other in vitro assays, like basophil activation, cytokines, chemokines and cellular markers have also been 
used for the study of AIT. ${ }^{17}$ The detection of basophil degranulation markers (mainly CD63 and CD203c) can be used to detect the suppression of basophil activation induced by SCIT. ${ }^{17}$ Changes in markers associated with dendritic cells, such as the upregulation of complement subunit $\mathrm{C} 1 \mathrm{q}$ expressed at the surface of regulatory DCs or downregulation of the DC2-associated CD141, are examples of promising biomarkers for the monitoring of SLIT's efficacy. ${ }^{17,31}$

Studies that investigate in vitro markers of AIT are progressively elucidating the mechanisms of AIT. Efforts are done to discover the appropriate biomarkers, reflecting allergy symptoms. Their future use would monitor immunologic changes directly connected with clinical outcomes. Although huge steps have been performed in the field of allergen immunotherapy, it seems that there is still a gap between the development of immunological biomarkers and their meaningful clinical use.

\section{In vivo Biomarkers}

A late-phase effect of AIT is the decrease in skin test reactivity, reflecting the reduce of tissue mast cells and eosinophils. ${ }^{16}$ AIT-induced reduction in skin reactivity has been estimated either by reduced size of SPT's wheal, or by an increased threshold for positive SPT reaction to the use of different allergen concentrations. ${ }^{32-35}$ Although reduction in SPT reactivity is a common outcome in AIT studies, its correlation to change of symptom scores is rarely mentioned. ${ }^{33,34}$

Since the performance of SPT is a mandatory step of allergy's diagnostic procedure and is part of Allergist's daily routine, its potential use as an in vivo follow-up biomarker, detecting AIT's efficacy, would be very practical. However, clinical experience and research findings suggest that skin testing does not represent a good marker or predictor of clinical symptoms; SPT is rather an "indicator" of allergen sensitization and not always of actual allergy. ${ }^{36}$ Upon completing an as-indicated-long AIT by performing SPT we may detect a decrease in sensitization to the allergens that have been used; however, these endpoints have to be interpreted only as such and not as clinical endpoints.

Allergy provocation tests are definitely consisting an indispensable diagnostic tool in various fields of allergy, posing diagnosis in cases that sensitization and clinical history are discordant. ${ }^{37}$ In the case of rhinitis, nasal allergy provocation tests (NAPT) are available for daily practice. They can set diagnosis in cases of discrepancies between symptoms and SPT/sIgE outcomes, in local allergic rhinitis that may present with negative sIgE and/or SPT and emerge the culprit allergen AIT in cases of allergen multisensitivity. ${ }^{37,38}$ NAPT are reproducing the response of the upper airways to natural allergen exposure under controlled and reproducible conditions. ${ }^{12}$ A positive NAPT detects the threshold dose that triggers nasal symptoms during the administration of escalating allergen doses. During NAPT, peak nasal inspiratory flow measurement is used to detect congestion, while the use of a Visual Analog Scale (VAS) is usually used for the evaluation of other nasal symptoms. ${ }^{37}$

The use of NAPT is a decision-making tool before AIT of polysensitized patients since it can detect the burden of each allergen in inducing the relative symptoms. ${ }^{37}$ NAPT have been used in several clinical trials on different types of AIT. ${ }^{12}$ Thanks to high sensitivity and specificity, NAPT is a reliable diagnostic tool, although it is time-consuming, needs training to perform it and readiness to face probable reactions like an asthma attack. The conjunctival provocation test is a reliable alternative of NAPT and a high correlation between them has been documented. ${ }^{39}$

Similar to NAPT but more sophisticated and considered lightly more accurate is the environmental challenge chamber (ECC) method. ${ }^{40}$ ECC reproduces the environmental conditions of pollen season and this method is used not only in clinical trials of AIT but also to evaluate antiallergic drugs. ${ }^{41-43}$ NAPT and ECC induce clinical symptoms that differ in magnitude and kinetics, but similar immunologic responses. ${ }^{40}$ The fact that NAPT can be widely used without need of chamber settings, overcomes the advantages of ECC, at least for use in the daily practice.

\section{Clinical Outcome Measures}

In the process of AIT vaccine development and approval, clinical trials are conducted, focusing on treatment's efficacy and safety. The reduction in symptoms and - the inseparably connected - decreased use of antiallergic drugs are considered the primary clinical endpoints of AIT clinical trials; however, a strict definition of clinical responders in AIT trials is still under discussion. ${ }^{6,15,44}$ QoL and a positive impact on work or studying (when students are regarded) are also fields that AIT aims to improve and are considered as secondary endpoints in clinical trials. $^{6}$

In order to monitor any of these parameters connected to AIT's efficacy, relevant scores or values have to be defined. 
The scores/values that reflect the parameters of AIT under consideration have to be evaluated and registered before the start of AIT, as well as during and at the completion of the treatment. These values or scores have to be reproducible and easy to use for the population under study. Comparison of scores during the process of AIT can detect alterations in clinical symptoms and offer data of clinical endpoints. In order to design measurement of health-related patientreported outcomes a reliable methodology has to be followed. On this purpose the Consensus-based Standards for the selection of health status Measurement Instruments (COSMIN) checklist has been developed. ${ }^{45}$

Symptom scores are the most common clinical values used in AIT studies and they are calculated following various methods. In the Guideline on the clinical development of products for specific immunotherapy of EMA as well as in FDA's Guidance, a patient self-rating method for the estimation of symptom scores is suggested. ${ }^{15,46}$ According to this method, four signs and symptoms of rhinitis (rhinorrhea, nasal itching, sneezing, congestion) plus two of conjunctivitis (itching/grittiness/redness and ocular tearing) are rated, with the use of a 4-point rating scale (Table 1). The use of the $0-3$ rating scale for each of the six signs/symptoms sums up to a total of $0-18$. This rhinoconjunctivitis total symptom score (RTSS) can be extracted on a daily basis.

In order to get the get RTSS data, patients have to be trained on how to self-assess symptomatology and registration. Symptom score should be registered at the same time of the day, for example, during the intake of SLIT drops or during breakfast for patients receiving subcutaneous AIT. In order to make clinical comparison before, during and after AIT, RTSS registration has to start before the beginning of AIT, setting the baseline of patients' symptomatology.

The extraction and use of a mean total rhinoconjunctivitis symptom score (RCSS) out of the daily scores, representing symptoms during a month's period or during

Table I A 4-Point Rating Scale to Rate Each Symptom/Sign of Rhinoconjunctivitis

\begin{tabular}{|l|l|}
\hline $\mathbf{0}$ & Absence of signs or symptoms \\
\hline $\mathbf{I}$ & Mild symptoms that can easily be tolerated \\
\hline $\mathbf{2}$ & Moderate symptoms that are bothersome but tolerable \\
\hline $\mathbf{3}$ & $\begin{array}{l}\text { Severe symptoms hard to tolerate, causing interference with } \\
\text { activities of daily living and/or sleeping }\end{array}$ \\
\hline
\end{tabular}

pollen season, seems more practical. ${ }^{4}$ For subjects allergic to pollen it is necessary to register baseline and endpoint symptom scores on and off pollen season; this way symptoms' severity can be defined and offer information on AIT's efficacy comparing average RTSS before the start and after the completion of immunotherapy. ${ }^{6}$ There are certainly difficulties in combining RCSS registration with the level of pollen exposure and comparison of symptom scores drives to safe results only if it refers to seasonal periods and geographical areas with similar pollen counts. ${ }^{47}$ For AIT to house dust mites or animal allergens comparison of RTSS in different treatment phases must also refer to similar levels of allergen exposure. ${ }^{6}$

RCSS can also be used beyond clinical trials. Allergists can apply it in everyday practice and suggest their patients to keep a diary of RTSS in order to follow-up the effects of AIT. However, as often described, adherence to keeping a diary is not always satisfying, so patients need continuous motivation and reminders. ${ }^{48}$ It is unrealistic to ask patients to keep a dairy during all 3-year long period, but easier to ask them to do it for a short period on and off pollen season.

Upper and lower airways are closely associated in health as well as in disease, so many patients with allergic rhinitis present concomitant asthma. AIT aims to prevent asthma and/or to control the entire allergic inflammation of the respiratory system. ${ }^{49}$ When a patient under AIT suffers from allergic rhinitis and concomitant asthma it is clear that asthma symptoms must be included in the score symptom monitoring. Extending the abovementioned method of the 0-3 scale for the calculation of daily symptom scores, AIT studies on patients with allergic rhinoconjunctivitis and asthma have rated symptoms in various combinations. Some of them have evaluated 3 (rhinorrhea, sneezing, congestion) or 4 (previous + nasal itching) rhinitis symptoms, 3 conjunctivitis symptoms (itching, redness, ocular tearing) and 3 symptoms of asthma (cough, wheezing, dyspnea). ${ }^{12}$

Offering different evaluation of symptoms in patients with allergic rhinoconjunctivitis and asthma, the Control of Allergic Rhinitis and Asthma Test (CARAT) questionnaire has also been developed. CARAT meets 9 of the 10 items on the COSMIN checklist and is composed of 10 questions that address symptoms of allergic rhinoconjunctivitis and asthma, sleep interference, activity limitation and the need to increase medication over 4 weeks. ${ }^{47}$ Answers are - similarly to the scale described above rated on a four-point scale. CARATkids is the version for 
children composed of 17 questions with "Yes" and "No" as answering options. ${ }^{50}$ CARAT and CARATkids are proposed to be used both in clinical trials and clinical practice.

Aiming to the optimal treatment of our patients, antiallergic medications are not stopped as soon as AIT starts, but instead pharmaceutical treatment is adapted to patients' current symptoms. Patients under subcutaneous AIT have the advantage of regular visits, so instructions on pharmacotherapy can be renewed frequently, aiming to minimize symptoms with minimal drug use. In clinical trials rescue medication is also permitted and a patient can use them, as needed, for bothersome symptoms. ${ }^{15}$

There is an interaction between symptom scores and medication scores; the use of antiallergic therapy ameliorates symptoms, while on the other hand the decrease of symptoms after AIT is translated to less antiallergic therapy. A balanced and validated scoring system is needed to register the combined symptom and medication score. ${ }^{6,12}$ A daily symptom score (dSS) combined with a daily medication score (dMS) has been suggested by an EAACI Task Force. ${ }^{6}$ In order to obtain the dSS, RTSS (allowing a maximum score of 18) is calculated and divided by 6 (number of symptoms). This calculation offers a dSS score range of 0 to 3 . On the other had, dMS is extracted from the medications used daily to ameliorate symptoms and also ranges from 0 to 3 . Combined Symptom Medication Score is the sum of dSS+dMS (range 0-6).

The needs for monitoring clinical outcomes in clinical practice are different than in clinical trials. A practical, fast, easy-to-use scoring tool is more likely going to be used by the patient and interpreted by the physician. Visual analogue score (VAS) is an easy self-assessment method, that may be used for the assessment of the rhinoconjunctivitis discomfort. Using a $10-\mathrm{cm}$ vertical scale, patients are pointing a number that best represents the severity of their symptoms, grading from 0 for "no symptoms" to 10 for "highest level of symptoms". 51

VAS can be used to assess every single symptom or to grade patients' global discomfort, even retrospectively (eg, during last day or in the last week). The use of mHealth apps, like the MASK-Air can make VAS registration of nose, eye and asthma symptoms easier and help to the adherence of patients in keeping a dairy. ${ }^{52}$ VAS is fully validated in adults and - although not validated for children - can also be used for pediatric patients. ${ }^{53}$

Questionnaires on QoL have also been developed and used as instruments in clinical trials. Rhinoconjunctivitis
Quality of Life Questionnaire (RQLQ), is one of these, measuring the more troublesome functional impairments that are affecting patient's social, emotional and physical well-being. ${ }^{54}$ RQLQ is composed of 28 questions in 7 domains (activity limitation, sleep problems, nose symptoms, eye symptoms, non nose/eye symptoms, practical problems and emotional function) and patients are using it to recall symptom severity during last week, grading on a 7-point scale. ${ }^{54,55}$ RQLQ has been validated and there is a variation of it for use by children and adolescents. ${ }^{56}$

Other questionnaires assessing health-related QoL that have been used for allergic rhinoconjunctivitis and asthma are the generic SF-36 Health Status Questionnaire and the disease-specific Rhinasthma. ${ }^{57,58}$ They can be very accurate to describe the effect of AIT on various parameters regarding respiratory allergy. However, a disadvantage in using these extensive questionnaires is that they demand more time than VAS or combined symptom-medication score. Patients are invited to answer 28 (RQLQ, Rhinasthma) to 36 questions (SF-36), which means that more that 10 minutes are needed each time. ${ }^{55,57,58}$ Concluding extended QoL questionnaires are unpractical for use other than Clinical Trials.

Finally, it is worth mentioning the registration of symptom-free or "well" days that has also been used to evaluate the effect of AIT. The idea is to compare the number of days with bothering symptoms and need for rescue medications before and after AIT. This is a measurement of clinical outcomes that can be easily applied in daily practice. ${ }^{12,15}$ However, it is a "grosso modo" measurement and parameters such as differences in allergenic load between two compared therapeutical periods, or the definition of symptoms' threshold that make a day "bad", have to be kept in mind when "symptom-free days" are evaluated.

\section{Practical Issues}

Combined symptom-medication score seems appropriate as a measurement tool of clinical outcomes. ${ }^{6}$ In adults, self-assessment of symptoms and signs and their registration is required. When children are under investigation, parents or caregivers are the ones evaluating and registering the symptom score, but also deciding on the use of rescue medication. Personality characteristics may interfere with the evaluation of symptom/sign severity or the decision to take rescue medication. Similarly, in the case of children, medication use may not directly represent the level of their symptoms' severity. However, this 
"subjective effect" will rather affect all registration periods of each patient, so combined symptom-medication score remains a valuable tool.

In order to evaluate the way that a patient is using a self-rating tool, after a short training, it is useful to compare actual clinical symptoms to patient's grading. Assessment of the data registered by a patient at the start and comparison with the ones at a second time during AIT can confirm how reliable the score's use is for each patient. This practice can be applied to both children and adults. Of course, patient's willingness to keep a dairy is a prerequisite and doctor's contribution with motivation and reminders in each medical visit is helpful.

Although in clinical trials it is mandatory to use a validated tool in order to confirm a product's efficacy, there is no need to use strictly validated tools to monitor our patients' clinical symptoms in everyday practice. VAS is an example of a tool that, although not validated for children, is so easy to use that it can be extremely helpful as a tool of clinical follow-up. VAS can be used in parallel with symptom and medication scores and when improvement is registered in all measures a safer conclusion is offered. $^{59}$

Although there are ethical issues for the use of mHealth tools by children and adolescents (for example, electronic on-line registration of VAS) and in some countries this is a problem that remains to be solved, mHealth tools are extremely helpful for the registration of scores in all ages. ${ }^{11}$ It is particularly useful in children since the use of technology is very attractive, and increased adherence to filling an electronic dairy in a cellphone app guarantees higher adherence than filling a printed form.

An allergist may choose any tool of preference and it is strongly suggested to make comparisons on symptom scores and rescue medicines using the same tool throughout AIT treatment. It is also advisable to use the same tool for all AIT-patients in a single Allergy office or an Outpatient Clinic, making their training easier and avoiding mistakes of follow-up. The use of such a tool to monitor our patients' clinical outcomes contributes to tailor-made decisions on changing an AIT treatment (ie, changing product) in cases of unsatisfying clinical result, prolonging or completing AIT, confirming that a 3-yearlong AIT has been successful.

\section{Disclosure}

The author reports no conflicts of interest in this work.

\section{References}

1. Bousquet J, Khaltaev N, Cruz AA, et al. Allergic rhinitis and its impact on asthma. J Allergy Clin Immunol. 2001;108:S147-S334.

2. Nurmatov U, Dhami S, Arasi S, et al. Allergen immunotherapy for allergic rhinoconjunctivitis: a systematic overview of systematic reviews. Clin Transl Allergy. 2017;7(1):24. doi:10.1186/s13601-0170159-6

3. Dhami S, Zaman H, Varga EM, et al. Allergen immunotherapy for insect venom allergy: a systematic review and meta-analysis. Allergy. 2017;72(3):342-365. doi:10.1111/all.13077

4. Frankland AW, Augustin R. Prophylaxis of summer hay-fever and asthma: a controlled trial comparing crude grass-pollen extracts with the isolated main protein component. Lancet. 1954;263 (6821):1055-1057. doi:10.1016/S0140-6736(54)91620-7

5. Frati F, Incorvaia C, Lombardi C, Senna G. Allergen immunotherapy: 100years, but it does not look like. Eur Ann Clin Immunol. 2012;44:99-106.

6. Pfaar O, Demoly P. Recommendations for the standardization of clinical outcomes used in allergen immunotherapy trials for allergic rhinoconjunctivitis: an EAACI position paper. Allergy. 2014;69:854-867.

7. Bousquet PJ, Brozek J, Bachert C, et al. The CONSORT statement checklist in allergen-specific immunotherapy: a GA2LEN paper. Allergy. 2009;64(12):1737-1745. doi:10.1111/j.1398-9995.2009. 02232.x

8. Roberts G, Pfaar O, Akdis CA, Ansotegui IJ, Durham SR. EAACI guidelines on allergen immunotherapy: allergic rhinoconjunctivitis. Allergy. 2018;73:765-798.

9. Senti G, von Moos S, Tay F, et al. Epicutaneous allergen-specific immunotherapy ameliorates grass pollen-induced rhinoconjunctivitis: a double-blind, placebo-controlled dose escalation study. J Allergy Clin Immunol. 2012;129(1):128-135. doi:10.1016/j.jaci.2011.08.036

10. Witten M, Malling HJ, Blom L, Poulsen BC, Poulsen LK. Is intralymphatic immunotherapy ready for clinical use in patients with grass pollen allergy? J Allergy Clin Immunol. 2013;132(5):1248-1252. doi:10.1016/j.jaci.2013.07.033

11. Matricardi PM, Dramburg S, Alvarez-Perea A, Antonin-Amerigo D, Apfelbacher C. The role of mobile health technologies in allergy care: an EAACI position paper. Allergy. 2020;75(2):259-272. doi:10.1111/all.13953

12. Pfaar O, Kleine-Tebbe J, Hörmann K, Klimek L. Allergen-specific immunotherapy: which outcome measures are useful in monitoring clinical trials? Immunol Allergy Clin North Am. 2011;31(2):289-309. doi:10.1016/j.iac.2011.02.004

13. Kouser L, Kappen J, Walton RP, Shamji MH. Update on biomarkers to monitor clinical efficacy response during and post treatment in allergen immunotherapy. Curr Treat Options Allergy. 2017;4 (1):43-53. doi:10.1007/s40521-017-0117-5

14. Atkinson AJ, Colburn WA, DeGruttola VG, DeMets DL, Downing GJ, Hoth DF. Biomarkers and surrogate endpoints: preferred definitions and conceptual framework. Clin Pharmacol Ther. 2001;69:89-95.

15. EMA-Committee for medical products for human use. Guideline on the Clinical Development of Products for Specific Immunotherapy for the Treatment of Allergic Diseases. London, Doc. Ref. CHMP/EWP/ 18504/2006. 2008.

16. Akdis M, Akdis C. Mechanisms of allergen-specific immunotherapy: multiple suppressor factors at work in immune tolerance to allergens. $J$ Allergy Clin Immunol. 2014;133(3):621-631. doi:10.1016/j. jaci.2013.12.1088

17. Shamji MH, Kappen JH, Akdis M, et al. Biomarkers for monitoring clinical efficacy of allergen immunotherapy for allergin rhinoconjunctivitis and allergic asthma: an EAACI position paper. Allergy. 2017;72(8):1156-1173. doi:10.1111/all.13138 
18. Di Lorenzo PA, Mansueto P, Pacor ML, et al. Evaluation of serum $\mathrm{s}-\mathrm{IgE} /$ total IgE ratio in predicting clinical response to allergen specific immunotherapy. $J$ Allergy Clin Immunol. 2009;123 (5):1103-1110. doi:10.1016/j.jaci.2009.02.012

19. Jutel M, Jaeger L, Suck R, Meyer H, Math D, Fiebig H. Allergenspecific immunotherapy with recombinant grass pollen allergens. $J$ Allergy Clin Immunol. 2005;116(3):608-613. doi:10.1016/j. jaci.2005.06.004

20. Shamji MH, Ljørring C, Francis JN, et al. Functional rather than immunoreactive levels of IgG4 correlate closely with clinical response to grass pollen immunotherapy. Allergy. 2012;67 (2):217-226. doi:10.1111/j.1398-9995.2011.02745.x

21. Gomez E, Fernandez TD, Dona I, et al. Initial immunological changes as predictors for house dust mite immunotherapy response. Clin Exp Allergy. 2015;45(10):1542-1553. doi:10.1111/cea.12578

22. Gehlar K, Schlaak M, Becker WM, Bufe A. Monitoring allergen immunotherapy of pollen-allergic patients: the ratio of allergen-specific IgG4 to $\operatorname{IgG} 1$ correlates with clinical outcome. Clin Exp Allergy. 1999;29(4):497-506. doi:10.1046/j.13652222.1999.00525.x

23. James LK, Shamji MH, Walker SM, et al. Long-term tolerance after allergen immunotherapy is accompanied by selective persistence of blocking antibodies. J Allergy Clin Immunol. 2011;127(2):509-516. doi:10.1016/j.jaci.2010.12.1080

24. James LK, Till SJ. Potential mechanisms for IgG4 inhibition of immediate hypersensitivity reactions. Curr Allergy Asthma Rep. 2016;16(3):23. doi:10.1007/s11882-016-0600-2

25. Baron-Bodo V, Horiot S, Lautrette A, et al. Heterogeneity of antibody responses among clinical responders during grass pollen sublingual immunotherapy. Clin Exp Allergy. 2013;43(12):1362-1373. doi:10.1111/cea.12187

26. Zenner HP, Baumgarten C, Rasp G, Kunkel G, Hauswald B, Ring J. Short-term immunotherapy: a prospective, randomized, double-blind, placebo-controlled multicenter study of molecular standardized grass and rye allergens in patients with grass pollen-induced rhinitis. J Allergy Clin Immunol. 1997;100(1):23-29. doi:10.1016/S00916749(97)70190-8

27. Van Neerven RJJ, Knol EF, Ejraes A, Wrtzen PA. IgE-mediated allergen presentation and blocking antibodies: regulation of T-cell activation in allergy. Int Arch Allergy Immunol. 2006;141 (2):119-129. doi:10.1159/000094714

28. Wurtzen PA, Lund G, Lund K, Arvidsson M, Rak S, Ipsen H. A double-blind placebo-controlled birch allergy vaccination study II: correlation between inhibition of IgE binding, histamine release and facilitated allergen presentation. Clin Exp Allergy. 2008;38 (8):1290-1301. doi:10.1111/j.1365-2222.2008.03020.x

29. Petersen AB, Gudmann P, Mikvang-Gronager P, et al. Performance evaluation of a specific IgE assay developed for the ADVIA centaur immunoassay system. Clin Biochem. 2004;37(10):882-892. doi:10.1016/j.clinbiochem.2004.06.010

30. Shamji MH, Wilcock LK, Wachholz PA, et al. The IgE-facilitated allergen binding (FAB assay): validation of a novel flow-cytometric based method for the detection of inhibitory antibody responses. $J$ Immunol Methods. 2006;317(1-2):71-79. doi:10.1016/j. jim.2006.09.004

31. Gueguen C, Bouley J, Moussu H, et al. Changes in markers associated with dendritic cells driving the differentiation of either $\mathrm{TH} 2$ cells or regulatory $\mathrm{T}$ cells correlate with clinical benefit during allergen immunotherapy. J Allergy Clin Immunol. 2016;137(2):545-558. doi:10.1016/j.jaci.2015.09.015

32. Kjellman NIM, Lanner A. Hyposensitization in childhood hay fever. A comparison of refined and whole timothy extracts. Allergy. 1980;35 (4):323-334. doi:10.1111/j.1398-9995.1980.tb01774.x

33. Østerballe O. Immunotherapy in hay fever with two major allergens 19,25 and partially purified extract of timothy grass pollen. Allergy. 1980;35(6):473-489. doi:10.1111/j.1398-9995.1980.tb01795.x
34. Bousquet J, Maasch H, Martinot B, Hejjaoui A, Wahl R, Michel FB. Double-blind, placebo-controlled immunotherapy with mixed grass-pollen allergoids. II. comparison between parameters assessing the efficacy of immunotherapy. J Allergy Clin Immunol. 1988;82 (3):439-446. doi:10.1016/0091-6749(88)90017-6

35. Sun W, Pan L, Yu Q. The skin prick test response after allergen immunotherapy in different levels of tIgE children with mite sensitive asthma/rhinitis in south China. Hum Vaccin Immunother. 2018;14 (10):2510-2515. doi:10.1080/21645515.2018.1482171

36. Bowton DL, Fasano MB, Bass DA. Skin sensitivity to allergen does not accurately predict airway response to allergen. Ann Allergy Asthma Immunol. 1998;80(2):207-1143. doi:10.1016/S10811206(10)62957-2

37. Agache I, Bilò BM, Braunstahl G-J, et al. In vivo diagnosis of allergic diseases-allergen provocation tests. Allergy. 2015;70 (4):355-365. doi:10.1111/all.12586

38. Campo P, Barrionuevo E, Eguiluz I, Salas M, Torres J, Rondón C. Nasal provocation tests with allergens: just a research tool or suitable for everyday clinical practice? Curr Treat Options Allergy. 2017;4 (1):98-109. doi:10.1007/s40521-017-0118-4

39. Richelmann H, Epple B, Gropper G. Comparison of conjunctival and nasal provocation test in allergen rhinitis to house dust mite. Int Arch Allergy Immunol. 2003;130(1):51-59. doi:10.1159/000068369

40. Larson D, Patel P, Salapatek AM, et al. Nasal allergen challenge and environmental exposure chamber challenge: a randomized trial comparing clinical and biological responses to cat allergen. J Allergy Clin Immunol. 2020;145(6):1585-1597. doi:10.1016/j.jaci.2020.02.024

41. Day JH, Briscoe M, Widlitz MD. Cetirizine, loratadine, or placebo in subjects with seasonal allergic rhinitis: effects after controlled ragweed pollen challenge in an environmental exposure unit. $J$ Allergy Clin Immunol. 1998;101(5):638-645. doi:10.1016/S0091-6749(98) 70172-1

42. Day JH, Briosce MP, Rafeiro E, Ellis AK, Pettersson E, Åkerlund A. Onset of action of intranasal budesonide (Rhinocort aqua) in seasonal allergic rhinitis studied in a controlled exposure model. J Allergy Clin Immunol. 2000;105(3):489-494. doi:10.1067/mai.2000.104550

43. Horak F, Zieglmayer P, Zieglmayer R, et al. Early onset of action of a 5-grass-pollen 300-IR sublingual immunotherapy tablet evaluated in an allergen challenge champer. J Allergy Clin Immunol. 2009;124 (3):471-477. doi:10.1016/j.jaci.2009.06.006

44. Pfaar O, Alvaro M, Cardona V, Hamelmann E, Mösges R, KleineTebbe J. Clinical trials in allergen immunotherapy: current concepts and future needs. Allergy. 2018;73(9):1775-1783. doi:10.1111/ all.13429

45. Mokkink LB, Terwee CB, Knol DL, et al. The COSMIN checklist for evaluating the methodological quality of studies on measurement properties: a clarification of its content. BMC Med Res Methodol. 2010;10(1):22. doi:10.1186/1471-2288-10-22

46. U.S. Department of Health and Human Services, FDA Food and Drug Evaluation and Research (CDER). Guidance for Industry. Allergic Rhinitis: Clinical Development Programs for Drug Products. Draft Guidance. 2000.

47. Amar SM, Harbeck RJ, Sills M, Silveira LJ, O' Brien H, Nelson HS. Response to sublingual immunotherapy with grass pollen extract: monotherapy versus combination in a multiallergen extract. Allergy Clin Immunol. 2009;124(1):150-156. doi:10.1016/j.jaci.2009.04.037

48. Pitsios C, Dietis N. Ways to increase adherence to allergen immunotherapy. Curr Med Res Opin. 2019;35(6):1027-1031. doi:10.1080/03007995.2018.1552044

49. Halken S, Larenas-Linnemann D, Roberts G, et al. EAACI guidelines on allergen immunotherapy: prevention of allergy. Pediatr Allergy Immunol. 2017;28(8):728-745. doi:10.1111/pai.12807

50. Tosca MA, Del Barba P, Licari A, Ciprandi G, Asthma Rhinitis Control Study Group. The measurement of asthma and allergic rhinitis control in children and adolescents. Children (Basil). 2020;7 (5):43. doi:10.3390/children7050043 
51. Canonica GW, Baena-Cagnani CE, Bousquet J, Bousquet PJ, Lockey RF, Malling HJ. Recommendations for standardization of clinical trials with allergen specific immunotherapy for respiratory allergy. A statement of a world allergy organization (WAO) taskforce. Allergy. 2007;62(3):317-324. doi:10.1111/j.1398-9995.2006.01312.x

52. Bousquet J, Hellings PW, Agache I, et al. ARIA 2016: care pathways implementing emerging technologies for predictive medicine in rhinitis and asthma across the life cycle. Clin Transl Allergy. 2016;6:47.

53. Bousquet PJ, Combescure C, Klossek JM, Daures JP, Bousquet J. Change in visual analogue score in a pragmatic randomized cluster trial of allergic rhinitis. J Allergy Clin Immunol. 2009;123 (6):1349-1354. doi:10.1016/j.jaci.2009.02.033

54. Juniper EF, Guyatt GH. Development and testing of a new measure of health status for clinical trials in rhinoconjunctivitis. Clin Exp Allergy. 1991;21(1):77-83. doi:10.1111/j.1365-2222.1991.tb00807.x

55. Juniper EF, Thompson AK, Ferrie PJ, Roberts JN. Validation of a standardised version of the rhinoconjunctivitis quality of life questionnaire. $J$ Allergy Clin Immunol. 1999;104(2):364-369. doi:10.1016/S0091-6749(99)70380-5
56. Juniper EF, Howland WC, Roberts NB, Thompson AK, King DR. Measuring quality of life in children with rhinoconjunctivitis. J Allergy Clin Immunol. 1998;101(2):163-170. doi:10.1016/S00916749(98)70380-X

57. Brazier JE, Harper R, Jones NM, et al. Validating the SF-36 health survey questionnaire: new outcome measure for primary care. $B M J$. 1992;305(6846):160-164. doi:10.1136/bmj.305.6846.160

58. Baiardini I, Pasquali M, Giardini A, et al. Rhinasthma: a new specific QoL questionnaire for patients with rhinitis and asthma. Allergy. 2003;58(4):289-294. doi:10.1034/j.1398-9995.2003.00079.x

59. Eifan AO, Akkoc T, Yildiz A, et al. Clinical efficacy and immunological mechanisms of sublingual and subcutaneous immunotherapy in asthmatic/rhinitis children sensitized to house dust mite: an open randomized controlled trial. Clin Exp Allergy. 2010;40(6):922-932. doi:10.1111/j.1365-2222.2009.03448.x

\section{Publish your work in this journal}

The Journal of Asthma and Allergy is an international, peer-reviewed open-access journal publishing original research, reports, editorials and commentaries on the following topics: Asthma; Pulmonary physiology; Asthma related clinical health; Clinical immunology and the immunological basis of disease; Pharmacological interventions and new therapies. The manuscript management system is completely online and includes a very quick and fair peer-review system, which is all easy to use. Visit http://www.dovepress.com/testimonials.php to read real quotes from published authors. 